\title{
Neck Lymph Node Groups
}

National Cancer Institute

\section{Source}

National Cancer Institute. Neck Lymph Node Groups. NCI Thesaurus. Code C132507.

Lymph node groups found within the seven levels and sublevels of the neck. (AJCC 8th ed.) 